\title{
Teamwork and Patient Education as Tools to the Reduce Overuse of Antibiotics - An Interview Study with Nursing Students at Hanoi Medical University
}

\author{
Kristin Andersen Ljungdahl, RN, BSc, Frida Swedenfeldt, RN, BSC and Kristina \\ Rosengren, $P h D, R^{*}$
}

Sahlgrenska Academy, Institute of Health and Care Sciences, University of Gothenburg, Sweden

*Corresponding author: Kristina Rosengren, $P h D, R N$, Associate Professor, Sahlgrenska Academy, Institute of Health and Care Sciences, University of Gothenburg, PO Box 457, SE 40530 Gothenburg, Sweden, Tel: +46-31-7866959, ORCID iD: 0000-0002-3221-2062

\begin{abstract}
The overuse of antibiotics due to poor health and suffering is a threat to global health. In Vietnam, overuse is a widespread problem because of the high availability of antibiotics and lack of knowledge about their use, and nurses have a significant role in informing and helping patients experience higher levels of health and well-being. The aim of this study was to describe Vietnamese nursing students' experience with antibiotics in healthcare. A qualitative content analysis with an inductive approach was used based on six semistructured interviews with nursing students at Hanoi Medical University in Vietnam. The results formed one category, "Uncertainty regarding antibiotic use", and three subcategories: "To obtain limited knowledge", "To contribute to improved knowledge", and "To manage selfmedication without the support of a health professional". Limited knowledge influences health professionals as well as patients and causes poor health, which contributes to the nonprofessional use of antibiotics. In conclusion, increased learning regarding antibiotics (e.g., evidencebased knowledge) as well as pedagogic skills and a sense of coherence (SOC) can be used to reduce the overuse of antibiotics, and interprofessional health education can facilitate patient education and professional teamwork.
\end{abstract}

\section{Keywords}

Antibiotics, Nursing student, Patient education, Qualitative content analysis, SOC

\section{Introduction}

The discovery of antibiotics has had positive effects, saving lives by preventing and curing widespread infections worldwide. However, overuse of antibiotics has resulted in antibiotic resistance, a threat to global health through bacterial infections, such as pneumonia, tuberculosis and sepsis, that become difficult to treat or, in some cases, impossible to treat due to resistance to antibiotics [1]. Therefore, nurses' promotion of health is significant in limiting unnecessary antibiotic treatment, which is why the present study intends to broaden the knowledge area by studying the experience of forthcoming nurses (i.e., nursing students) with antibiotics.

\section{Background}

Antibiotics are crucial to reducing illness and death caused by severe infections that are, for example, associated with surgery, intensive care and chemotherapy [1]. However, to maintain efficacy, it is important to use narrow-spectrum antibiotics related to the reason for treatment (e.g., bacterial infection) to reduce new infections due to a weakened immune system [2]. In addition, a contributing factor is that antibiotics are available prescription-free in a number of countries [3], which is why self-medication without the assessment of healthcare professionals is common in Vietnam [4]. The increased use of antibiotics results in bacteria adapting, which is why antibiotic resistance is constantly evolving worldwide. Resistant bacteria develop by enzymatic modification breakdown, which reduces antibiotic uptake through the cell wall. Resistance leads to longer 
hospital stays, higher costs and increased mortality [1]. In addition, the development of new antibiotics does not progress at the same rate that the old antibiotics lose their effects [5]. By 2050, 10 million people are estimated to die from untreated infections due to antibiotic resistance resulting from the overuse of antibiotics [6].

According to the WHO [1], improvements are required at the individual, organizational and community levels due to unsuitable selling of antibiotics without a prescription, as are guidelines for the prescription of antibiotics [4]. Furthermore, citizens need education on antibiotics and their consequences [1], which is why nurses have a significant role in improving knowledge and skills by teaching patients and their relatives to prevent poor health [7]. Promoting health to prevent disease and relieving suffering from a salutogenic perspective could be described by the three parts of a SOC (sense of coherence): Comprehensibility, meaningfulness and manageability [8]. The degree of the SOC is determined by the individual's ability to handle and relate to different situations; for example, nurses' health promotion affects patients' experiences of a SOC. Good communication, i.e., interactions between nurses and patients built upon information and counseling, is used to understand patients' health situations as comprehensible, meaningful and manageable [9]. A sense of coherence is expressed when patients and their relatives participate in healthcare [8], which is why relationships (e.g., trust and respect) among nurses, patients and relatives are crucial to improving knowledge and understanding the caring situation as being meaningful [9].

According to Rankin, et al. [10], patient education is an essential component of optimizing patient health status by influencing his/her attitude and understanding regarding the care situation. The indications of antibiotics, as well as their mechanisms of action, effects and side effects, are important information for increasing patients' and relatives' awareness regarding the treatment of poor health [2]. Nurses have the significant task of influencing patients' self-care through pain management and the use of antibiotics through patient education [11]. Nurses who share information, demonstrating genuine commitment to patient questions with active listening, increase patient motivation to participate in healthcare [8]. Interaction within patient education increases the possibilities for patients to achieve a high level of a SOC [9], with results such as decreased suffering as well as improved health and well-being [7].

The Vietnamese healthcare system includes both public and private providers at four levels: central, provincial, district and municipal care [12]. Approximately 90 percent of antibiotic sales are made without prescriptions or consultation with health professionals and are used in self-medication for coughs and fevers as well as the prevention of poor health. These practices result in the overuse of antibiotics because patients wish to avoid costly and time-consuming consultations with health professionals [4]. Moreover, antibiotic use in Vietnam differs between rural and urban areas; people in urban areas have higher economic status and education levels, which results in critical thinking related to the use of antibiotics compared to the populations of rural areas [4]. Furthermore, Vietnam has limited resources, as well as old equipment and overcrowded hospitals where patients share beds - increasing the risk of infection, which also increases the use of antibiotics and complicates nurses' abilities to prevent poor health [13]. In addition, nursing education programs vary in length (1-5 years) and a lack of standardized curricula results in differences in theoretical knowledge and practical skills among Vietnamese nurses. Therefore, guidelines for the curriculum for nursing education have been developed by the Vietnamese government; however, each nursing school can change parts of the curriculum, which is why quality in nursing education can differ [13].

In summary, the overuse of antibiotics is a growing worldwide problem that results in antibiotic resistance. In Vietnam, antibiotics can be purchased without a prescription, which results in extensive self-medication due to a lack of resources as well as limited knowledge regarding antibiotics. Therefore, nurses have an important role in improving knowledge through patient education to prevent poor health; therefore, forthcoming nurses need knowledge and skills regarding antibiotics through high-quality nursing education.

Aim

The aim of this study was to describe Vietnamese nursing students' experiences with antibiotics in healthcare.

\section{Method}

\section{Setting}

The study was conducted at Hanoi Medical University (HMU), the oldest and largest university in Vietnam, offering high-quality education and research in healthcare $[14,15]$. HMU offers education to a total of 10,000 students (at undergraduate and postgraduate levels); 500 of them are nursing students, and $50 \%$ of them ( $n=250$ ) attend the advanced nursing program (4.5 years) taught in English. These nursing students have theoretical classes/courses during morning sessions and clinical placement in the afternoons at, for example, Hanoi Medical University Hospital.

\section{Design}

This study used manifest qualitative content analysis with an inductive approach to reflect variations in nursing students' experiences with antibiotics in Vietnam. Qualitative methods were considered appropriate when data collection included individual interviews due to subjective perspectives. Content analysis was applied to create a deeper understanding and to draw conclusions from collected data $[16,17]$. 


\section{Sample}

The study used a strategic sample related to qualitative methods [18] to gather knowledge from a nursing student's perspective. Inclusion criteria for participation were nursing students who were in their final year of education at the bachelor's level for general nursing (in a 4.5-year advanced nursing program) at $\mathrm{HMU}$, who were over the age of 18 , who had experience with the use of antibiotics, and who were able to understand and speak English (to reduce linguistic misunderstandings). Prior to the data collection, two pilot interviews were carried out in Sweden to followup and improve the semi-structured interview guide as well as to test of technical equipment to achieve high quality of the audio recording. To facilitate data collection, information letters, consent forms and questions were translated from English to Vietnamese. Moreover, a contact teacher at HMU informed nursing students regarding current study's background, aim and methods, and two of the authors (KAL, FS) forwarded information letters and consent forms to interested students. The first six nursing students who were interested in participating and who fit the inclusion criteria were included in the study.

\section{Data collection}

Data were collected in October 2017, and all interviews $(n=6)$ took place in a room at HMU with two of the authors (KAL, FS). Before each interview began, written and oral information (in English and Vietnamese) were given, and signed consent forms (in English) were collected. None of the participants expressed a need for Vietnamese translation during the interviews. Semistructured interviews were conducted, which included background questions (e.g., age, education, experience in healthcare) and four perspectives regarding the use of antibiotics, educational content in nursing education, clinical experience, and future challenges regarding antibiotics. The interview began with, "If I say 'antibiotics', what you are thinking about"? and "Tell me about your experience with use of antibiotics in healthcare". Based on the answers, related questions were explored, and clarifications and further elaborations were conducted. Reformulation of questions was necessary in five out of six interviews to avoid misunderstandings due to English language (which was not their mother tongue), such as "Did I understand you right?", "Can you give an example?", and "Can you develop your answer?" All interviews were conducted individually (20-30 minutes), recorded and then transcribed verbatim. The participants ( 1 male,
5 female, ages 21-22) had experience in clinical practice in infection control, emergency care, surgery and internal medicine (semesters 5,6 , and 8 , respectively). In addition, three out of six participants had experience with voluntary work (1-24 months).

\section{Data analysis}

The interviews were analyzed using manifest qualitative content analysis $[16,17]$ to interpret the meaning from the content of the data and to address trustworthiness [18], with examples drawn from nursing students' experiences with use of antibiotics. Data analysis began with analysis of each interview (done by each individual author) to clarify the content that responded to the aim of the study. Thereafter, data $(n=6)$ were analyzed (by all authors) for meaning units, condensation, codes, subcategories and categories (Table 1). A manifest interpretation was used to describe the content of the written words - the basis for the analyses that were conducted in the following steps (Table 1): 1. Transcripts were read and reread to obtain an understanding of and familiarity with the text; 2. Meaning units (e.g., words, sentences or paragraphs) corresponding to the content areas were selected using an inductive approach concerning (a) Education and (b) Limited knowledge; 3. Each meaning unit was condensed into a description of its content and labeled with one of 255 codes; 4 . Subcategories were identified and grouped by their codes; and 5 . One category was identified: "Uncertainty regarding antibiotic use". Three subcategories were also created: "To obtain limited knowledge", "To contribute to improved knowledge", and "To manage self-medication without the support of a health professional".

The findings are illustrated with quotes.

\section{Ethical considerations}

Ethical approval and permission for the study were obtained from the dean of HMU. No ethical approval was required because of the Swedish rules and guidelines regarding student theses and/or quality improvement that had no negative effects for the participants $[19,20]$ (Swedish Code of Statutes, 1982:763; Codex 2017). However, ethical guidelines for human and social research were followed throughout the study: Information, informed consent, confidentiality and utility with no harm [20]. The information requirement was taken into account by giving the participants written and oral information about the aim of the study and data collection and about the interviews being recorded. All students were informed that

Table 1: Example of analysis and sorting of content into subcategories that formed a category.

\begin{tabular}{|l|l|l|l|l|}
\hline Meaning unit & Condensed content & Coding & Subcategory & Category \\
\hline $\begin{array}{l}\text { They choose to go to pharmacists } \\
\text { first, because the symptoms is... don't } \\
\text { seems all worst. They choose to go to a } \\
\text { pharmacy instead of going to a hospital. }\end{array}$ & $\begin{array}{l}\text { Pharmacists first, } \\
\text { symptoms doesn't } \\
\text { seems worst. Pharmacy } \\
\text { instead hospital. }\end{array}$ & $\begin{array}{l}\text { Self-medication } \\
\text { in manage self- }\end{array}$ & $\begin{array}{l}\text { Uncertainty } \\
\text { medication without the } \\
\text { regarding antibiotic } \\
\text { support of a health }\end{array}$ & use \\
\hline
\end{tabular}


participation was voluntary, that they had the right to withdraw at any time and that their answers would be kept confidential; no names were used, and findings are described in categories without identification. Moreover, information that students' participation/ avoidance of participation would not affect their study results was also given.

\section{Findings}

The results formed one category, "Uncertainty regarding antibiotic use", and three subcategories, "To obtain limited knowledge", "To contribute to improved knowledge", and "To manage self-medication without the support of a health professional". Limited knowledge influences health professionals as well as patients and causes poor health, which contributes to the nonprofessional use of antibiotics (Figure 1).

\section{Uncertainty regarding the use of antibiotics}

A feeling of uncertainty regarding the use of antibiotics was experienced by the nursing students and other health professions, such as nurses, physicians and pharmacists, as well as patients and their relatives. The nursing students highlighted that uncertainty arises during nursing education related to limited theoretical knowledge (few teacher-led lessons) and practical skills (limited tasks). Participants described that the above limitations resulted in uncertainty in terms of prescription requirements and the management and administration of antibiotics. Furthermore, participants noted increased suffering from the patients' and relatives' perspectives because staff within healthcare do not provide information that is evidence-based. This results in insufficient support and assistance regarding illness, which contributes to increased, nonprofessional self-medication based on the patient's and relatives' experiences. According to the nursing students, selfmedication occurs because of uncertainty regarding the use of antibiotics and is without compliance regarding evidence-based knowledge of treatments, timing and doses. Moreover, participants highlighted that the overuse of antibiotics within Vietnam's healthcare system, as well as nonprofessional self-medication within the population, leads to antibiotic resistance. They argued that this problem is a threat to health and well-being in Vietnam, as well as the rest of the world, and that it requires improvement efforts at the individual and community levels.

\section{To obtain limited knowledge}

To obtain limited knowledge is described as insufficient knowledge regarding antibiotics and their impacts on health and well-being. Participants described that their nursing education had two theoretical lessons (in pharmacology) in which antibiotics were part of the teaching content that focused on the medical aspects of antibiotics. According to the nursing students, limited knowledge influenced their patient education, resulting in insufficient information regarding antibiotic practices that prevent antibiotic resistance. In addition, the

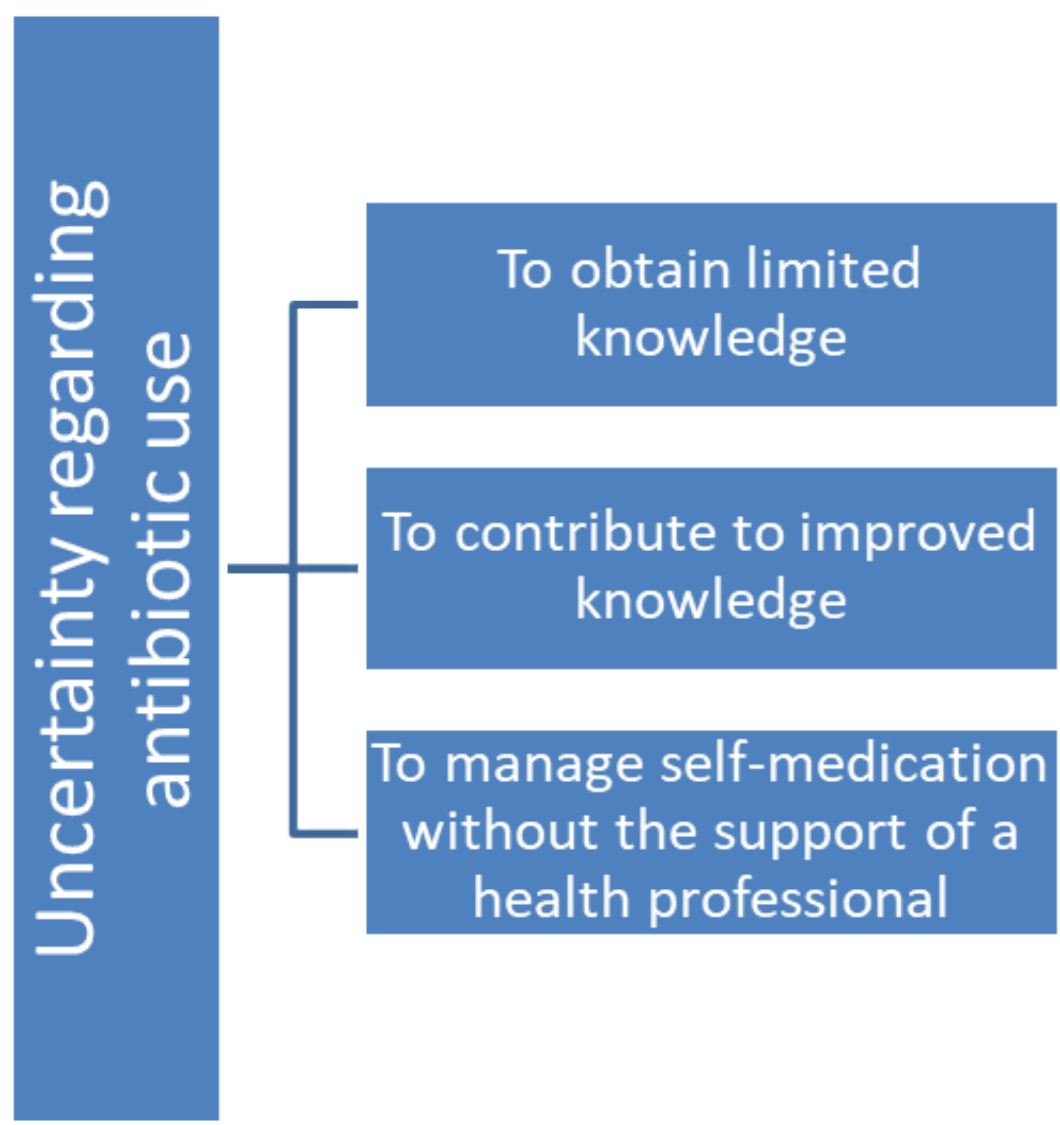

Figure 1: The category and subcategories generated in the results. 
nursing students emphasized improvement regarding education from a global perspective to increase evidence-based knowledge within the problem areas of antibiotics. Furthermore, the students stressed difficulties in applying their limited knowledge in clinical settings, i.e., it is difficult to remember theoretical knowledge from nursing education, and the management and administration of antibiotics varies by clinical placement. The nursing students described uncertainty in providing antibiotics at the right time in relation to side effects, including mechanisms of action related to specific diseases and/or poor health. Participants also highlighted that limited knowledge forces them to be careful about the management and administration of antibiotics and stressed that improved evidence-based knowledge would decrease the use of antibiotics.

Participants argued that counseling from a nursing perspective is limited or even nonexistent regarding antibiotic-related issues due to the hierarchical healthcare organization and high workloads. The nursing students noted nurses' limited time (10-22 patients/nurse) for providing patient education (e.g., support or counseling), especially when most of the patients do not ask for this kind of nursing. Participants highlighted the need to reduce the number of patients per nurse as well as to use instruction/guidelines with evidence-based content on indications, management and administration, possible side effects and advice regarding antibiotics. They suggested that the receipt of information associated with antibiotic use must be acknowledged with a signed form before treatment begins:

Patients need to know all of the information about antibiotics, yeah. And have a form, like a form, and the patient can sign their signature if they agree to, for this kind of antibiotic or not. After they know about the names of antibiotics, the, what is usefulfor the antibiotic and side effects of antibiotics when they choose this or not. I think we need to have a form, to ask the patient if they agree or disagree. (5)

The nursing students described that they improved their limited knowledge using available sources such as the internet, books and newspapers. They argued that independent learning parallel to curriculum within nursing education is important, alongside independent repetition to update their knowledge; otherwise, the information can be easily forgotten. Limited knowledge was gained by observing how nurses and physicians deal with patient behavior as well as perform practical tasks such as managing antibiotics. In addition, the nursing students participated in external learning possibilities at HMU as well as compulsory courses in nursing education and teaching sessions in hospital settings based on their clinical placements - for example, a seminar on antibiotic resistance.
Moreover, the nursing students described limited knowledge among health professionals working in hospital settings, which resulted in overuse and further antibiotic resistance, a major problem in Vietnam. The nursing students highlighted the importance of extended opportunities for conferences and meetings to spread knowledge about antibiotic resistance among health professionals to improve healthcare. They highlighted that limited knowledge in smaller hospitals may be one reason for the overuse of antibiotics. Nursing students noted that patients who are transferred to larger hospitals had therefore already developed resistance to several antibiotics. Another issue is overloaded and crowed hospitals that have up to three patients sharing a single bed, which is why infections are widespread and antibiotics are frequently used. According to the nursing students, the quality and content of nonpharmacological counseling varies, which highlights the need for nurses to include patient education in their daily work to decrease the use of antibiotics for poor health. Another perspective described by the nursing students was that patients receive many medications during hospital care, including antibiotics.

\section{To contribute to improved knowledge}

Contributing to improved knowledge regarding antibiotics requires information about the experiences of different health professional roles, such as physicians and pharmacists. According to the participants, the physician always prescribes antibiotics; however, the nursing students stress that prescriptions are not always based on clinical examination regarding symptoms and in accordance with evidence-based medicine. The students stressed that physicians do not always prescribe the correct antibiotic, stating the following:

When I come to the hospital now I can see that most of the patients, um, even, even though, even those who don't have infections, the, they receive the prescription from the doctor and it includes an antibiotic too. (6)

Furthermore, the nursing students also highlighted that physicians prescribe strong antibiotics, such as vancomycin, which is not in line with evidence-based knowledge. The students have learned that nurses are entitled to discuss evidence-based knowledge with physicians regarding issues such as the use of antibiotics; however, they usually do not choose this option. One reason for this is that in Vietnam's culture, physicians are powerful in the field of healthcare compared to nurses; therefore, discussion regarding drug treatment with physicians is rare and they do not always listen to the nurses' thoughts or views. Furthermore, the nursing students pointed out uncertainty about the guidelines for antibiotics as a reason for why nurses might inform a patient about side effects without specifying how the side effects could be managed. The students also highlighted that patients are not always informed about 
antibiotic resistance in relation to the prescription from the physician. Prior to drug administration, a nurse's responsibility is to discuss side effects in light of a patient's background, status and further treatment. The nursing students emphasized that nurses ask the physicians about side effects; however, as forthcoming nurses, they stress the significance of discussing patients' treatments to prevent the improper prescription of antibiotics following admission to the hospital. Moreover, the nursing students also expressed a wish to work more independently to handle antibiotics in a patient-safe manner and in line with evidence-based knowledge.

According to the nursing students, pharmacists provide limited patient information at the time an antibiotic is purchased. The nursing students stressed that pharmacists working in a pharmacy focus on sales, so they provide simple advice, such as taking antibiotics with meals, when patients directly ask for information instead of providing correct information regarding the administration of the drug and the drug's side effects.

I think it is a problem because the patients don't have information on the drugs they use. 'Cause the pharmacists just, they just want to sell drugs, medications. That's why, when the patient orders something, they just give them. And, you know, a lot of pharmacists they don't inform, don't inform the patients about side effects of the, this drugs and how to use them correctly. Some patient they like overdose, use overdose drugs. (1)

Pharmacists working in pharmacies have an impact on antibiotic use in Vietnam. The nursing students described their doubts regarding pharmacists' evidence-based knowledge on supporting patients with proper antibiotic treatment. One reason the students highlighted is the short education required for pharmacists, which is why their knowledge seems to be experience-based instead of evidence-based:

I know that some pharmacists... they study six months and they give antibiotic for the patient and they don't know anything, they just kind of listen to some symptoms that the patient has. (3)

To manage self-medication without the support of a health professional

The students' solutions for managing selfmedication without the support of a health professional included the improvement of patient education as a part of nurses' daily work to increase evidence-based strategies for self-medication involving antibiotics. The nursing students highlighted that patient information from health professionals (e.g., nurses, physicians, pharmacists) is medically oriented with limited room for adaptation from the patient's perspective. The students stressed a lack of information regarding drug names and indications as well as correct timing, doses and durations, which should be a natural role for them, as nurses are educated in medical universities on how to improve professional self-medication. Moreover, nursing students pointed out that informing patients about activities such as having healthy eating habits, resting and/or getting physical exercise is an essential part of nursing and patient education for influencing patients' daily lives, as well as the lives of their families and friends, i.e., Nurses can improve knowledge and awareness within the population to increase health and well-being to avoid health issues that require antibiotics.

Patient self-medication strategies affect the overuse of antibiotics in Vietnam. A possible reason raised by the nursing students for this is limited awareness within the population about antibiotic use and its consequences, which affects attitudes towards antibiotics in relation to health conditions. The students stressed that people self-diagnosis and self-medicate themselves with antibiotics for colds with flu-like symptoms, such as coughs, fevers, skin rashes, constipation and diarrhea, instead of receiving support from health professionals. In addition, some households have antibiotics available at home from previous treatments that are then used for symptoms described above. Furthermore, the nursing students reported that people receive information about self-medication from the internet and/or verbal recommendations from friends and family members, which is why self-medication with antibiotics instead of seeking professional counseling and support regarding self-medication is common in Vietnam. Problems with self-medication of antibiotics is more common in rural areas that are further from hospitals. Patients and relatives' habitual purchasing of antibiotics at the pharmacy without a prescription could be related to Vietnam's culture as well as being based on previous experiences (of the individual or family). The nursing students argued that using self-medication is considered comfortable and cheap compared to visiting a crowded health clinic or hospital that is located far away charges a fee for treatment. In addition, long waiting times before consultation, examination, diagnosis and treatment, as well as patients being required to read and sign multiple documents at the hospital, contribute to increased selfcare without health professional support. Furthermore, the fear of meeting physicians and the risk of other diseases influence patients' choices for self-medication without health professional support, which explains why the nursing students stressed insufficient compliance with antibiotic treatments regarding timing and doses.

For example, the pharmacy gives them doses for five days. Usually like that. So, but they just keep take the medications for like three days or two days, like they see symptoms decrease or disappear. So they stop, they think they get over it. (2)

The nursing students pointed out that above experience-based self-medication and lack of compliance with treatment standards lead to resistance, which explains why patients must self-medicate themselves with stron- 
ger antibiotics the next time. The students described that patients seek hospital care when self-medication has no effect on severe infections with mutating, multiresistant bacteria. The nursing students highlighted that antibiotics cannot cure all infections due to advanced resistance, which has resulted in a few deaths in Vietnam.

\section{One participant describes it as:}

I see some severe infectious disease, very, I am very afraid, because when we reach the history records of, of this patient, they say, they say that's, this person used too much of every antibiotic, so now we don't have any kinds of antibiotic to treat him. (5)

The nursing students described that there are rules and the population does not follow regulations in Vietnam regarding antibiotics. Laws for the limited use of antibiotics are considered as applicable to major cities; however, people in rural areas still buy antibiotics without prescriptions at local pharmacies. The nursing students emphasized the need for improvements that will to contribute to better health, such as only using prescriptions of antibiotics written by physicians, the use of national campaigns (on the TV, radio, and websites) directed towards all citizens in Vietnam regarding antibiotics and patient education performed by nurses when patients are in contact with healthcare professionals.

\section{Discussion}

The study results describe the nursing students' experiences regarding the use of antibiotics, as well as the attitudes regarding health and poor health, that influence the sense of uncertainty stated by the nursing students, which include limited knowledge of how to improve supported self-medication in their forthcoming roles as nurses. Limited knowledge influences health professionals as well as patients and results in poor health, which contributes to the overuse of antibiotics. Participants in the current study stressed the significance of educating patients and relatives to improve evidence-based self-medication related to poor health. However, to be prepared to perform patient education as a newly educated nurse, content in nursing education about antibiotics that is built on evidence-based knowledge must be improved. Adding a global perspective facilitates broader understanding regarding the overuse of antibiotics, which is a worldwide problem. By focusing on antibiotics in nursing education, forthcoming nurses could improve health promotion through patient education as a part of their daily work [21]. This conclusion is in line with Sherman [22], who stressed the importance of the nursing students receiving knowledge regarding patient information and education to increase health and wellbeing.

One factor related to the students' uncertainty is the lack of communication between nurses and physicians, which affects daily work and the quality of healthcare. Another influencing factor is the Vietnamese hierarchical healthcare structure, which contributes to a lack of teamwork and limited ability to ask questions and share knowledge [23]. Vat, et al. [24] highlighted that the quality of cooperation between health professions negatively influences patient safety. Hoving, et al. [25] described that patient information and education are affected by a lack of communication, which is why misunderstandings occur. Interprofessional learning (e.g., theoretical learning and simulation) is one way to improve communication, coordination and collaboration [26] between forthcoming health professionals, such as nurses, physicians and pharmacists, which will also improve the quality of healthcare by decreasing antibiotic resistance.

Furthermore, it appears that patients are not given enough information and/or are unable to ask questions due to the nurses' high workloads. This result is in line with Rankin, et al.'s [10] description that patients will not ask additional questions due to being unwilling to use the nurses' and physicians' limited time. Moreover, a stressful working environment influences the insufficiency of the conditions for patient education, which negatively affects nurses' tasks and promotion of health. In addition, Tervo-Heikkinen, et al. [27] argue that staffing, relationships between professionals and management support are factors that affect workload, which is why it is necessary to focus on teamwork to improve the working environment as well as patient safety.

In summary, the limited learning conditions, communication shortages and unfavorable working conditions mentioned above affect patient education. Limited knowledge and a lack of confidence and trust in healthcare can be seen as a few reasons that citizens manage self-medication without the support of a health professional, i.e., based on their own experiences instead of evidence-based knowledge. Research [28] shows that patient education supports patient involvement in healthcare. A well-informed, knowledgeable patient could prevent suffering and poor health due to lack of preparedness. Nurses who educate patients regarding diagnosis, treatment, possible complications and side effects increase overall health and well-being through health promotion. Patient education facilitates awareness among patients and motivates positive attitudes and knowledge at the individual and community levels [25]. Teaching and learning with two-way communication between nurses and patients involves facilitated partnerships, i.e., person-centered care [29].

Furthermore, participation facilitates empowerment when patients have the right conditions to ask questions in order to gain knowledge and understanding about 
antibiotics and their consequences [11]. Zakzesky, et al. [28] highlight that increased understanding is associated with a SOC, which strengthens patients' empowerment. Moreover, Rohrer, et al. [30] argue that well-informed patients control their own health by breaking old habits - for example, overuse of antibiotics by improved, evidence-based self-care. Patient education performed by well-educated nurses using evidencebased knowledge is a tool that can decrease the use of nonprofessional self-care. Knowledgeable patients can instead spread scientifically proven knowledge to friends and relatives to increase awareness regarding the overuse of antibiotics and its consequences among the population.

The results from the current study show that the nursing students are aware of today's overuse of antibiotics, and they have ambitions to improve the situation through teamwork as well as patient education. However, nursing education and knowledge among the Vietnamese population need to be improved. Moreover, improved teamwork with other health professionals, such as physicians and pharmacists, is also needed. By improving dialogues between different health professions as a team and counseling around different health conditions, increased awareness regarding antibiotic use could be developed among staff, patients and the relatives of patients. Future research areas could include the risks and effects of antibiotic use within healthcare from a nurse's perspective in order to promote health and prevent poor health at the individual and global levels.

\section{Limitations}

The study was conducted at one university in one country. This small sample limited the ability to generalize the results regarding how to decrease the overuse of antibiotics. However, trustworthiness of the results was ensured by a systematic scientific analysis, qualitative content analysis, and well-documented methodology for grasping people's experiences. The study's validity may also be questioned because the data collection was done with a limited number of participants. Furthermore, variation of the nursing students' experiences due to antibiotics could influence the use of results; therefore, more research is needed to contribute to the knowledge within nursing education regarding antibiotics, patient education and interprofessional teamwork.

\section{Conclusion}

In conclusion, increased learning regarding antibiotics and pedagogic skills within interprofessional health education facilitate the use of evidence-based knowledge in patient education as well as improve teamwork among health professionals, which, in turn, improves health and well-being. More work is necessary to improve and increase the knowledge and understand- ing of antibiotics and their consequences. This can be achieved through advanced nursing work, where a SOC can be used, i.e., a patient's experience of comprehensibility, manageability and meaningfulness provides conditions for reducing the overuse of antibiotics.

\section{Ethical Approval}

Ethical approval was not required for this study.

\section{Contributions}

Study design: KAL, FS, KR; Data collection KAL, FS; Data analysis KAL, FS, KR; Manuscript preparation; KR.

\section{Sources of Funding}

The authors received no financial support for this research or for authorship and/or publication of this article.

\section{Acknowledgements}

We thank the nursing students at Hanoi Medical University, Vietnam, who participated in the study, and the University of Gothenburg, Sweden.

\section{Conflicts of Interest}

The authors declared no conflicts of interest with respect to the authorship and/or publication of this article.

\section{References}

1. WHO (2018) Antibiotic resistance.

2. Crombie H (2012) Nurses can help prevent antibiotic resistance. Primary Health Care 22: 16-21.

3. Hollis A, Maybarduk P (2015) Antibiotic resistance is a tragedy of the commons that necessitates global cooperation. J Law Med Ethics 43: 33-37.

4. Nga DTT, Chuc NT, Hoa NP, Hoa NQ, Nguyen NT, et al. (2014) Antibiotic sales in rural and urban pharmacies in northern Vietnam: An observational study. BMC Pharmacol Toxicol 15: 6.

5. Cars O, Högberg L, Murray M, Nordbergq O, Sivaraman S, et al. (2008) Meeting the challenge of antibiotic resistance. BMJ 337

6. Dolgin E (2016) Inner workings: Combating antibiotic resistance from the ground up. Proc Natl Acad Sci U S A 113: 11642-11643.

7. International Council of Nurses (2014) The ICN code of ethics for nursing.

8. Antonovsky A (1987) Unraveling the Mystery of Health. Jossey-Bass Inc, San Francisco.

9. Fok SK, Chair SY, Lopez V (2005) Sense of coherence, coping and quality of life following a critical illness. J Adv Nurs 49: 173-181.

10. Rankin SH, Stallings KD, London F (2005) Patient education in health and illness. ( $5^{\text {th }}$ edn), Lippincott, New York.

11. Jotterand F, Amodio A, Elger BS (2016) Patient education as empowerment and self-rebiasing. Med Health Care Philos 19: 553-561.

12. Nguyen TK, Cheng TM (2014) Vietnam's health care 
system emphasizes prevention and pursues universal coverage. Health Aff (Millwood) 33: 2057-2063.

13. Nguyen LT, Annoussamy LC, Lebaron VT (2017) Challenges encountered by Vietnamese nurses when caring for patients with cancer. Oncol Nurs Forum 44: 147151.

14. Hanoi Medical University (2007) Organization: Hanoi Medical University.

15. Hanoi Medical University (2014) Introduction: Training capacity of Hanoi Medical University.

16. Graneheim UH, Lundman B (2004) Qualitative content analysis in nursing research: Concepts, procedures and measures to achieve trustworthiness. Nurse Educ Today 24: 105-112.

17. Graneheim UH, Lindgren BM, Lundman B (2017) Methodological challenges in qualitative content analysis: A discussion paper. Nurse Educ Today 56: 29-34.

18. Polit D, Beck CT (2017) Nursing research: Generating and assessing evidence for nursing practice. Wolters Kluwer Health/Lippincott Williams \& Wilkins, Philadelphia.

19. Health and Medical Care Act.

20. Codex (2017) Rules \& guidelines for research. The humanities and social sciences.

21. Löfmark A, Smide B, Wikblad K (2006) Competence of newly-graduated nurses - a comparison of the perceptions of qualified nurses and students. J Adv Nurs 53: 721-728.

22. Sherman JR (2016) An initiative to improve patient education by clinical nurses. Medsurg Nursing 25: 297-333.

23. Rice K, Zwarenstein M, Conn L, Kenaszchuk C, Russell A, et al. (2010) An intervention to improve interprofessional collaboration and communications: A comparative qualitative study. J Interprof Care 24: 350-361.

24. Vat M, Common C, Laizner AM, Borduas C, Maheu C (2015) Reasons for returning to the emergency department following discharge from an internal medicine unit: Perspectives of patients and the liaison nurse clinician. $J$ Clin Nurs 24: 3605-3614.

25. Hoving C, Adriaan V, Mullen Dolan P, van den Borne B (2010) A history of patient education by health professionals in Europe and North America: From authority to shared decision making education. Patient Educ Couns 78: 275-281.

26. Atack L, Parker K, Rocchi M, Maher J, Dryden T (2009) The impact of an online interprofessional course in disaster management competency and attitude towards interprofessional learning. J Interprof Care 23: 586-598.

27. Tervo-Heikkinen $T$, Partanen $P$, Vehviläinen-Julkunen K, Laaksonen K (2008) Working conditions of finnish registered nurses: A National Survey. Nordic Journal of Nursing Research 28: 8-17.

28. Zakzesky D, Klink K, McAndrew N, Schroeter K, Johnson G (2015) Bridges and barriers: Patients' perceptions of the discharge process including multidisciplinary rounds on a trauma unit. J Trauma Nurs 22: 232-239.

29. Ekman I, Swedberg K, Taft C, Lindseth A, Norberg A, et al. (2011) Person-centered care-ready for prime time. Eur J Cardiovasc Nurs 10: 248-251.

30. Rohrer J, Wilshusen L, Adamson SC, Merry S (2008) Patientcentredness, self-rated health, and patient empowerment: Should providers spend more time communicating with their patients? J Eval Clin Pract 14: 548-551. 